\title{
Anti-obesity effect of robusta fermented with Leuconostoc mesenteroides in high-fat diet-induced obese mice
}

\author{
SOO-YOUNG CHOI ${ }^{1}$, SEUNG-HYUN RYU ${ }^{1}$, JIN-IL PARK ${ }^{1}$, EUI-SUK JEONG ${ }^{1,2}$, \\ JONG-HYUNG PARK ${ }^{1}$, SEUNG-HOON HAM ${ }^{1}$, HEE-YEON JEON ${ }^{1}$, JUN-YOUNG KIM ${ }^{1}$, \\ INN-GOO KYEONG ${ }^{3}$, DONG-GOO KIM ${ }^{3}$, JI-YOUNG SHIN ${ }^{3}$ and YANG-KYU CHOI ${ }^{1}$ \\ ${ }^{1}$ Department of Laboratory Animal Medicine, College of Veterinary Medicine, Konkuk University, \\ Seoul 05029; ${ }^{2}$ Laboratory Animal Center, Daegu-Gyeongbuk Medical Innovation Foundation, \\ Daegu 41061; ${ }^{3}$ R\&D Center, CosisBio Corporation, Chungbuk 27867, Republic of Korea
}

Received February 27, 2017; Accepted August 2, 2017

\section{DOI: $10.3892 /$ etm.2017.4990}

\begin{abstract}
Robusta beans cultivated with Monascus ruber (RMR) were successively fermented with Leuconostoc mesenteroides (LM) and the antiobesity effects were examined. To produce an obese mouse model to investigate the hypolipidemic effects, ICR mice were fed the same high-fat diet for 6 weeks. Treatment groups were given 10 or $20 \%$ RMR-LM. Body weight changes in the $20 \%$ RMR-LM group were lower compared with those in the control group. Visceral adipose tissue weight and adipose size were significantly lower in the $20 \%$ RMR-LM group compared with those in the control group. Significant improvement in glucose tolerance was observed in the 10 and $20 \%$ RMR-LM groups compared with the control group. The $20 \%$ RMR-LM group exhibited a significant reduction in serum glucose concentration. Hepatic mRNA levels of sterol regulatory element-binding protein 1 , fas cell surface death receptor, and peroxisome proliferator-activated receptor $\gamma$, which are associated with lipid, and fatty acid metabolism, in the 20\% RMR-LM group were significantly lower compared with those in the control group. The results of the present study demonstrated that $20 \%$ RMR-LM may be used to prevent obesity, and ameliorate diabetes and lipid metabolism imbalances.
\end{abstract}

\section{Introduction}

Obesity is a medical condition characterized by increased adipose mass resulting from a chronic imbalance between energy intake and expenditure (1). In response to the increase

Correspondence to: Dr Yang-Kyu Choi, Department of Laboratory Animal Medicine, College of Veterinary Medicine, Konkuk University, 120 Neungdong-ro, Gwangjin-gu, Seoul 05029, Republic of Korea

E-mail: yangkyuc@konkuk.ac.kr

Key words: anti-obesity, high-fat diet, Leuconostoc mesenteroides, Monascus ruber, robusta in obesity, it has become increasingly more common to use pharmaceutical treatments for obesity, which are costly and not very effective $(2,3)$. Currently, there is a focus in anti-obesity treatments on alternative therapies, including natural products and herbal medicines. Functional, health-enhancing foods, or nutraceuticals, are food products that influence specific physiological functions in the body (4).

As the interest in natural products has increased, a microbial culture and fermentation products were used to develop food additives and healthy food supplements. Recently, the study of the physiological activities, including antioxidant activity and immune activation, of liquid mycelium cultures has progressed steadily (5). In addition, mycelium fermentation with natural products has the advantage of being able to utilize all of the useful natural components of the mycelium (5).

The natural product robusta have been reported to have various effects and physiological activities. Many herbal-derived polyphenolic compounds are suggested to be able to prevent obesity via hypolipidemia effects and adipose tissue reduction and thus suppress the development of the metabolic, hepatic, and cardiovascular alterations associated with obesity (6). Robusta had been shown to contain melanoidin, which has been shown to have antioxidant activity in vivo, and a large amount of chlorogenic acid, which is a functional material component (7). Yamabushitake mushroom (Hericium erinaceum) is a well-known edible and medical mushroom used in East Asia. It has been reported that $H$. erinaceum contains many biologically active compounds, which have shown interesting biological activities, such as hypolipidemic, cytotoxic, anti-microbial, and anti-tumor effects (8). Oral administration of dried Yamabushitake mushroom powder can improve mild cognitive impairment in humans, but little is known about the anti-obesity efficacy of H. erinaceum (9).

The ascomycetous fungus Monascus ruber has been used in food, medicine, and industry in Asian countries. The use of fungi for the production of commercially important products has increased rapidly over the past half century, and pigment-producing microorganisms and microalgae are quite common in nature (10). It has been reported that M. ruber produces monacolin $\mathrm{K}$, which has antifungal and 
immunosuppressive effects, and M.ruber is an effective treatment for hypercholesterolemia and cholesterol imbalances because it decreases blood cholesterol levels (11). A previous study on red mold rice production by Monascus species under monoculture conditions showed that secondary metabolite production is greatly affected by the fermentation medium, cultivation conditions, and types of Monascus species used in the fermentation process (12).

There is an increasing interest in the use of health functional materials, especially lactic acid bacteria (13). Leuconostoc mesenteroides is an epiphytic bacterium that is widely spread in the natural environment and used in the production of various useful products in the biochemical and pharmaceutical industries. In addition, it has been used to modify a variety of bioactive substances in an effort to improve their functionality.

After the primary fermentation of the mycelium of $M$. ruber with robusta as nutrients, the primary fermentation products were cultivated with L. mesenteroides in a secondary fermentation. It was expected that the two-step fermentation (Step-by-step) would enhance the physiological activity of the extracts, enabling their use in developing food additives and health supplements. The goal of this research was to investigate the effect of robusta fermented with L. mesenteroides on treating obesity.

\section{Materials and methods}

Preparation of first fermentation product. M. ruber were obtained from the National Institute of Agricultural Sciences (NAS, Jeollabuk-do, Korea) to ferment robusta. Briefly, the first seed cultivation of $M$. ruber were cultured on potato dextrose agar (PDA) for $10 \sim 15$ days at $25 \sim 30^{\circ} \mathrm{C}$ and then cultured in potato dextrose broth (PDB) for 4 7 days in a shaking incubator (Jeio Tech Co., DaeJeon, Korea). For robusta fermented with M. ruber (RMR) $100 \mathrm{~g}$ of Vietnam robusta bean was soaked in $200 \mathrm{ml}$ water for $2 \mathrm{~h}$, sterilized for $120 \mathrm{~min}$ at $121^{\circ} \mathrm{C}$, and allowed to cool down at room temperature. Robusta and $M$. ruber were mixed in ratio of $10: 1$, cultured for 7 days at $25^{\circ} \mathrm{C}$, and roasted after hot-air drying to produce the first solid fermented product.

Preparation of second fermentation product. Robusta cultivated with $M$. ruber (RMR) were secondarily fermented with Leuconostoc mesenteroides (RMR-LM). Briefly, L. mesenteroides a pure lactic acid bacteria, was separated from kimchi. Separated L. mesenteroides was transferred into Lactobacilli MRS agar and cultivated for $24 \mathrm{~h}$ at $30^{\circ} \mathrm{C}$ in a $\mathrm{CO}_{2}$ incubator (Sanyo Electric Co., Japan). The first fermentation products, glucose (cornstarch dextrose $100 \%$ ), L. mesenteroides, and water were mixed in ratio of 1:1:1:10 and cultured for $5 \sim 7$ days at $30^{\circ} \mathrm{C}$. When the cultivation was finished, the secondary fermentation products, RMR-LM, were extracted by hot water extraction. The extracts were filtered through $8 \mu \mathrm{l}$ filter paper and sterilized for $10 \mathrm{~min}$ at $100^{\circ} \mathrm{C}$ for use in experiments. The RMR-LM, the stock solution of each extract was defined as $100 \%$ extract. Extracts were diluted with sterilized water to examine the effects of different concentration of RMR-LM.
Animals and experimental designs. Male 5-week-old ICR mice (Hsd:ICR) were purchased from the Koateck (Gyeonggi-do, Korea) company. The mice were acclimatized for 1 week to allow adaptation, during which time they were fed a high-fat diet $(60 \%$ kcal as fat; D12492; Research Diet, Inc., New Brunswick, NJ, USA) to induce obesity. Each group of mice $(n=10)$, weighing between 28-34 g, was randomly divided and was allowed free access to the liquid extracts and a high-fat diet to maintain obesity for 6 weeks. Control mice had free access to water instead of liquid extracts. The groups were composed of the 10\% RMR-LM extract and 20\% RMR-LM extract groups. All mice were housed in a pathogen-free room under a constant 12 -h light-dark cycle at $22 \pm 2^{\circ} \mathrm{C}$ temperature and $50 \pm 10 \%$ humidity. Body weight and food intake were recorded once a week at the appointed time for 6 weeks. All of the animal experimental protocols were approved by the Institutional Animal Care and Use Committee of Konkuk University.

Intraperitoneal glucose tolerance test. For the glucose tolerance test, the mice were fasted for $6 \mathrm{~h}$ on the day before the final treatment, and the basal blood glucose levels ( $0 \mathrm{~min})$ were determined from the tail vein. Glucose was then intraperitoneally injected ( $1 \mathrm{~g} / \mathrm{kg}$ body weight), and additional blood glucose levels were measured at 15, 30, 60, and $120 \mathrm{~min}$ using a commercial glucometer (Accu-Chek Active; Roche, Mannheim, Germany). The area under the curve (AUC) for the glucose tolerance test was calculated.

Serum biochemical analysis. After $6 \mathrm{~h}$ of fasting, the mice were sacrificed, and blood samples drawn from the caudal vena cava for analysis. Serum glucose (GLU), triglyceride (TG), total cholesterol (TC), high-density lipoprotein cholesterol (HDL-c), low-density lipoprotein cholesterol (LDL-c), aspartate aminotransferase (AST), and alanine aminotransferase (ALT) levels were analyzed using a Hitachi 7020 Automatic Analyzer (Hitachi, Tokyo, Japan).

Histopathological analysis. The visceral adipose tissue (epididymal and retroperitoneal pad) was dissected, weighed, and fixed in $10 \%$ neutral buffered formalin solution. The sections were stained with hematoxylin and eosin (H\&E). The slides were examined under a BX51 light microscope (Olympus Corp., Tokyo, Japan). The adipocyte size in the adipose tissue was determined by dividing the number of the adipocytes by the total area counted.

Quantitative real-time PCR analysis. Total RNA was extracted from frozen liver using TRIzol (Ambion Inc., Austin, TX, USA) according to the manufacturer's instructions. The cDNA as a template was synthesized using M-MLV reverse transcriptase (Invitrogen Life Technologies, Merelbeke, Belgium). Gene expression was quantified using Real-Time PCR with a Bio-Rad CFX96 real-time PCR detection system (Bio-Rad, Mississauga, ON, Canada). The DNA primers for the target genes used in this study are listed in Table I. $\beta$-actin was used as an internal control for normalization.

Statistical analysis. All obtained data were analyzed using GraphPad Prism (GraphPad Software, Inc., La Jolla, CA, 
Table I. The primer sequences for the cDNAs of the housekeeping gene and target genes used in real-time PCR.

\begin{tabular}{lll}
\hline Gene & \multicolumn{1}{c}{ Forward primer } & \multicolumn{1}{c}{ Reverse primer } \\
\hline$\beta$-actin & 5'-AGCCTTCCTTCTTGGGTATGG-3' & 5'-CACTTGCGGTGCACGATGGAG-3' \\
FAS & 5'-AGGGGTCGACCTGGTCCTCA-3' & 5'-GCCATGCCCAGAGGGTGGTT-3' \\
PPAR $\gamma$ & 5'-CAAGAATACCAAAGTGCGATCAA-3' & 5'-GAGCTGGGTCTTTTCAGAATAATAAG-3' \\
LPL & 5'-CGCTCCATTCATCTCTTCA-3' & 5'-CTTGTTGATCTCATAGCCCA-3' \\
SREBP-1 & 5'-GGAGCCATGGATTGCACATT-3' & 5'-GGCCCGGGAAGTCACTGT-3' \\
ACC & 5'-GGAGATGTACGCTGACCGAGAA-3' & 5'-ACCCGACGCATGGTTTTCA-3' \\
\hline
\end{tabular}
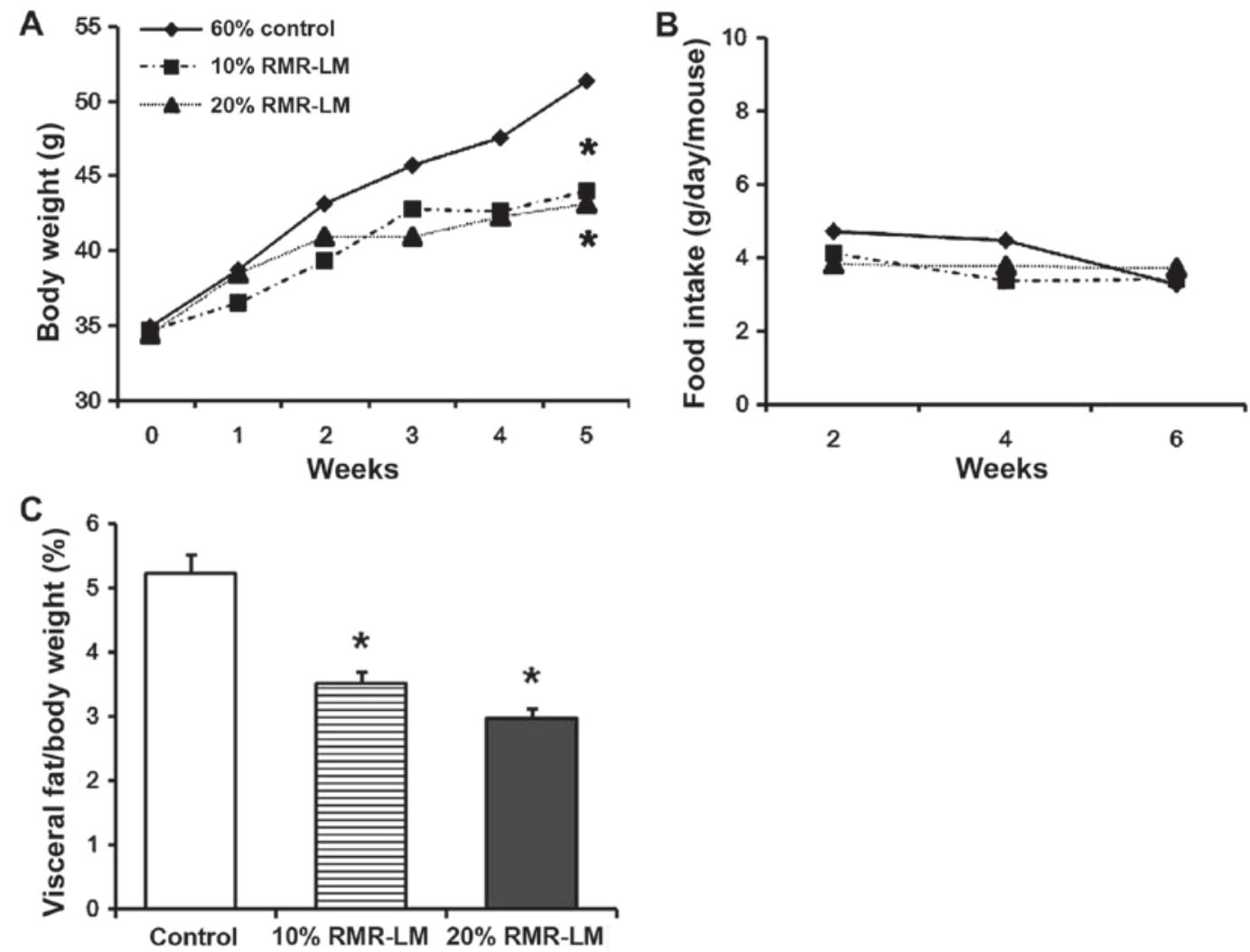

Figure 1. Body weight changes, average food intake levels, and total visceral fat weights. (A) Body weight changes; (B) Average food intake; (C) Total visceral fat/body weight. Average food intake calculated as $\mathrm{g} / \mathrm{day} /$ mouse. Data are expressed as the mean $\pm \mathrm{SD}$ ( $\mathrm{n}=10$ per group). ${ }^{*} \mathrm{P}<0.05$ vs. control.

USA) and expressed as the mean \pm standard deviation $\left({ }^{*} \mathrm{P}<0.05\right)$. Statistical significance was evaluated by one-way ANOVA with Dunnett's multiple comparison test. $\mathrm{P}<0.05$ was considered to indicate a statistically significant difference.

\section{Results}

Body weight changes, food intake levels, and total visceral fat weights. To examine the effects of RMR-LM concentration on anti-obesity, the mice were treated with distilled water (control group), 10\% RMR-LM and 20\% RMR-LM. The body weight gains of the $10 \%$ RMR-LM group and 20\% RMR-LM group were significantly $\left({ }^{*} \mathrm{P}<0.05\right)$ lower than that of the control group after 6 weeks (Fig. 1A). The food intake levels of all groups were not significantly different, although the body weight gain of the control group was significantly higher than those of the two RMR-LM groups (Fig. 1B). The effects of the extracts on visceral fat weight are shown in Fig. 1C. The total visceral fat weight decreased with increases in the dose of RMR-LM extract. Both the 10\% RMR-LM group and 20\% RMR-LM group has a significantly lower total visceral fat weight than the control group.

Adipocyte size in visceral adipose tissue. The number of adipocytes in the $20 \%$ RMR-LM group was significantly higher than in the control group (Fig. 2A), and as a result, the adipocyte size in the $20 \%$ RMR-LM group was significantly smaller than in the control group (Fig. 2B).

Antidiabetic effects determined by intraperitoneal glucose tolerance tests. To investigate the antidiabetic effects of RMR-LM, glucose tolerance tests were performed after 6 weeks of treatment as seen in Fig. 2. In the control group, the fasting blood glucose level increased following the injection 

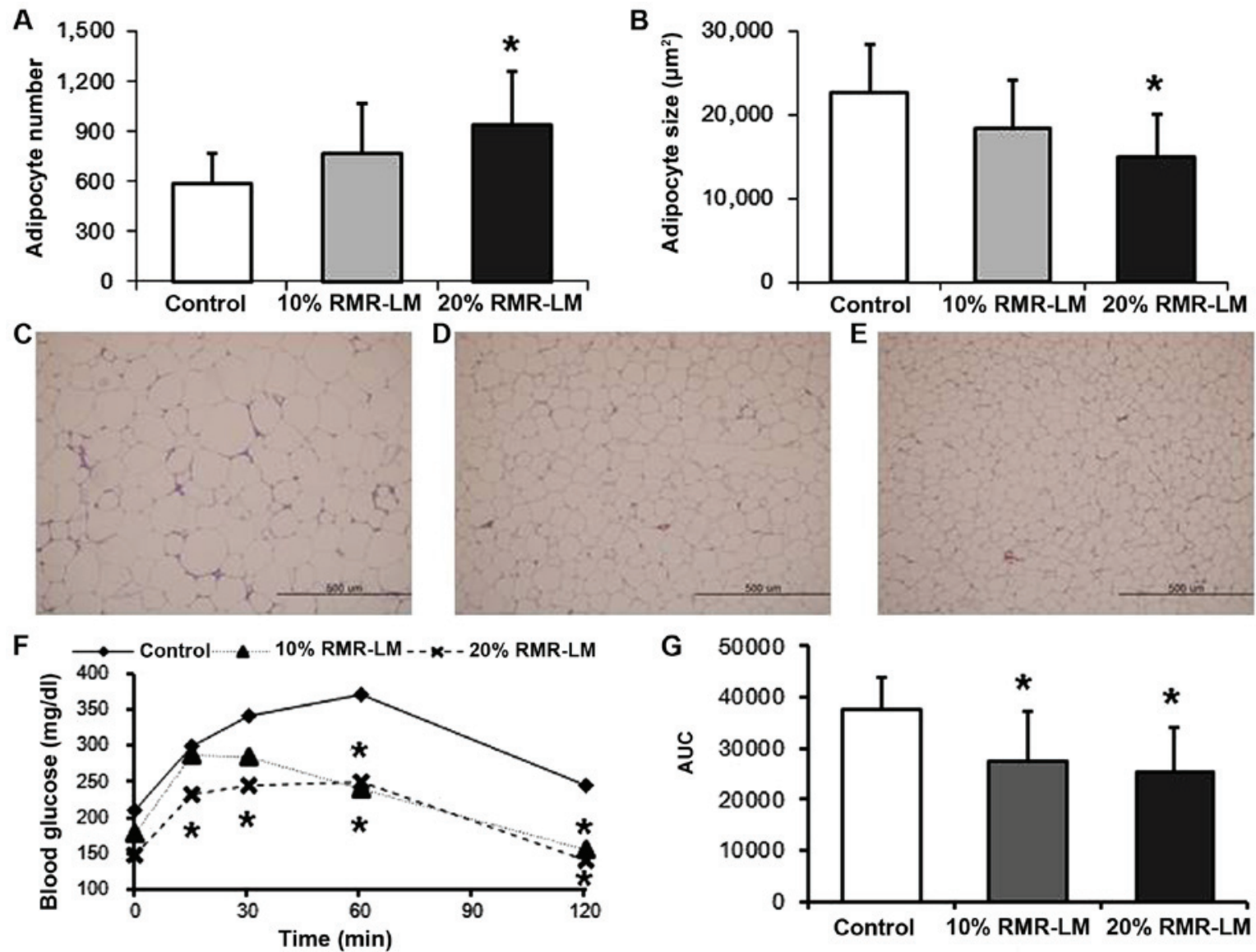

Figure 2. Adipocyte size in visceral adipose tissue intraperitoneal glucose tolerance tests. (A) Adipocyte number; (B) Adipocyte size; (C) Control of visceral adipose tissue, hematoxylin and eosin (H\&E) staining; (D) 10\% RMR-LM of visceral adipose tissue, H\&E staining; (E) 20\% RMR-LM of visceral adipose tissue, H\&E staining; (F) Intraperitoneal glucose tolerance test; (G) Area under the curve (AUC) of glucose tolerance test. Adipocyte size was calculated by dividing the number of adipocytes by the area counted. H\&E staining. Data are shown as the mean $\pm \mathrm{SD}\left(\mathrm{n}=10\right.$ per group). ${ }^{*} \mathrm{P}<0.05$ vs. control.

of glucose. The 20\% RMR-LM group showed significantly $\left({ }^{*} \mathrm{P}<0.05\right)$ improved glucose tolerance compared with the control group, indicating that the $20 \%$ RMR-LM treatment may improve glucose tolerance in obese mice. The blood glucose concentrations of the 10\% RMR-LM group at the 60- and 120-minute intervals were significantly lower than those of the control group. The AUC values for the glucose tolerance test of the 10\% RMR-LM group and 20\% RMR-LM group were significantly lower than that of the control group.

Serum biochemistry analysis of the lipid. The fasting serum TG and glucose levels were analyzed (Fig. 3A and B). The level of TG was not significantly different between the control group and the RMR-LM extract groups. The glucose level in the $20 \%$ RMR-LM group was significantly lower than that in the control group. The glucose level in the 10\% RMR-LM group was also lower than that of the control group, but this difference was not significant. The serum AST level of the $10 \%$ RMR-LM group was lower than that of the control group, but these differences were not significant. The serum ALT levels of the 10\% RMR-LM and 20\% RMR-LM groups were lower than that of the control group, but no significant difference was observed (Fig. 3C and D).

Gene expression analysis in the liver. The hepatic mRNA expression levels of genes related to lipid and fatty acid metabolism, including sSREBP-1, ACC, FAS, LPL, and PPAR $\gamma$, were analyzed by real-time PCR (Fig. 4). The mRNA expression levels of SREBP-1 in liver tissue in both the 10\% RMR-LM group and 20\% RMR-LM group were significantly $\left({ }^{*} \mathrm{P}<0.05\right)$ down-regulated compared with the control group, especially in the $20 \%$ RMR-LM group. The gene expression levels of FAS and ACC in the $20 \%$ RMR-LM group were significantly decreased compared with the control group. The PPAR $\gamma$ is a nuclear receptor that regulates adipocyte differentiation and liver lipid storage. The mRNA expression levels of PPAR $\gamma$ in liver tissue in both the 10\% RMR-LM group and 20\% RMR-LM group were significantly $\left({ }^{*} \mathrm{P}<0.05\right)$ down-regulated compared with the control group.

\section{Discussion}

In this study, the anti-obesity effects of fermented extracts on the development of obesity in ICR mice were examined. We selected successive two-step fermentation to enhance biological activity based on our data $(5,7)$. Robusta were fermented by successive two-step fermentation of M. ruber and L. mesenteroides (RMR-LM). The present results clearly show that the 20\% RMR-LM treatment is able to suppress diet-induced obesity. The effectiveness of the doses of RMR-LM $(10,20 \%)$ used in this study was validated by the significant body 

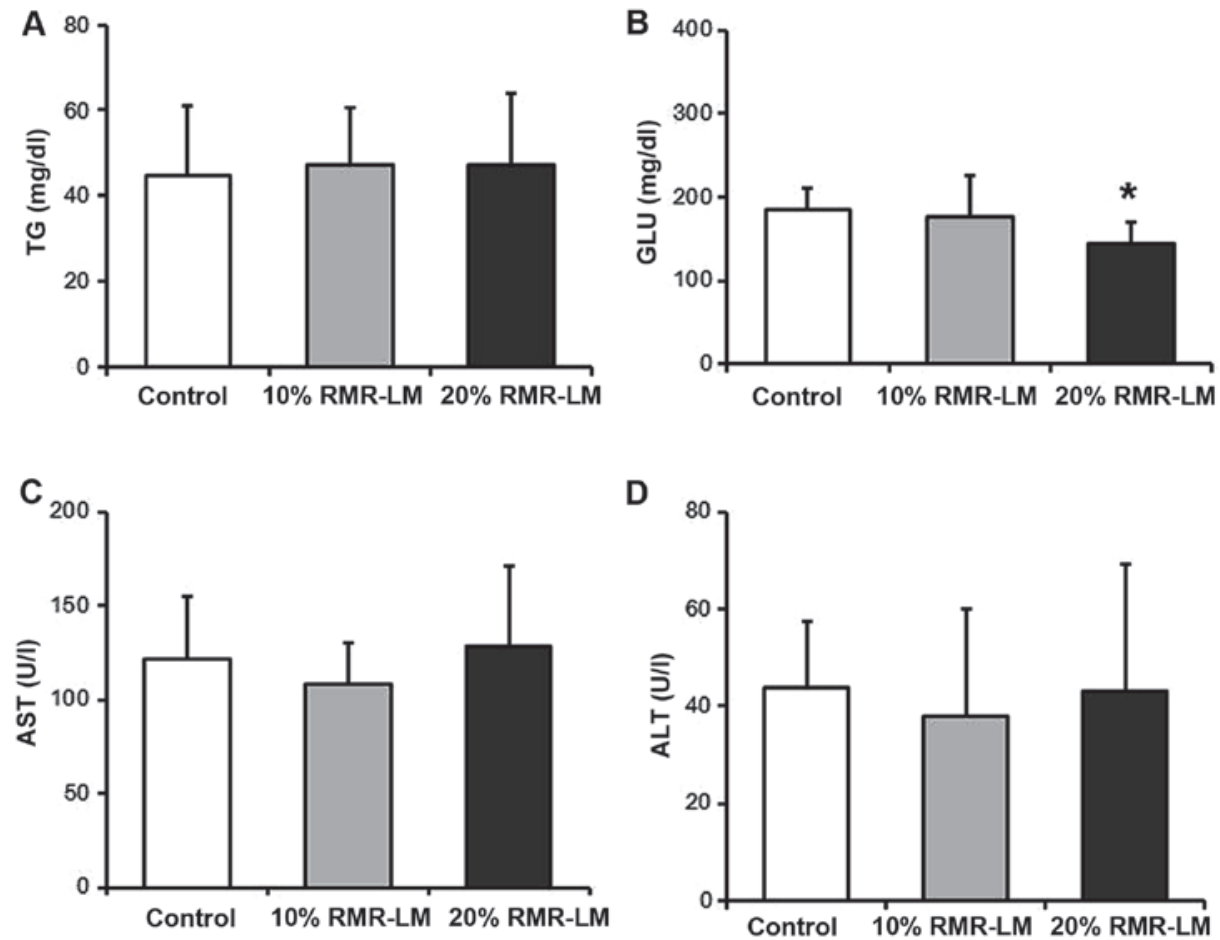

Figure 3. Effects on serum lipid and hepatic profiles by biochemistry analysis. (A) TG level; (B) GLU level; (C) AST level; (D) ALT level. Values are expressed as the mean \pm standard deviation ( $\mathrm{n}=10$ per group). " $\mathrm{P}<0.05$ vs. control. TG, triglyceride; GLU, glucose; AST, aspartate aminotransferase; ALT, alanine aminotransferase.
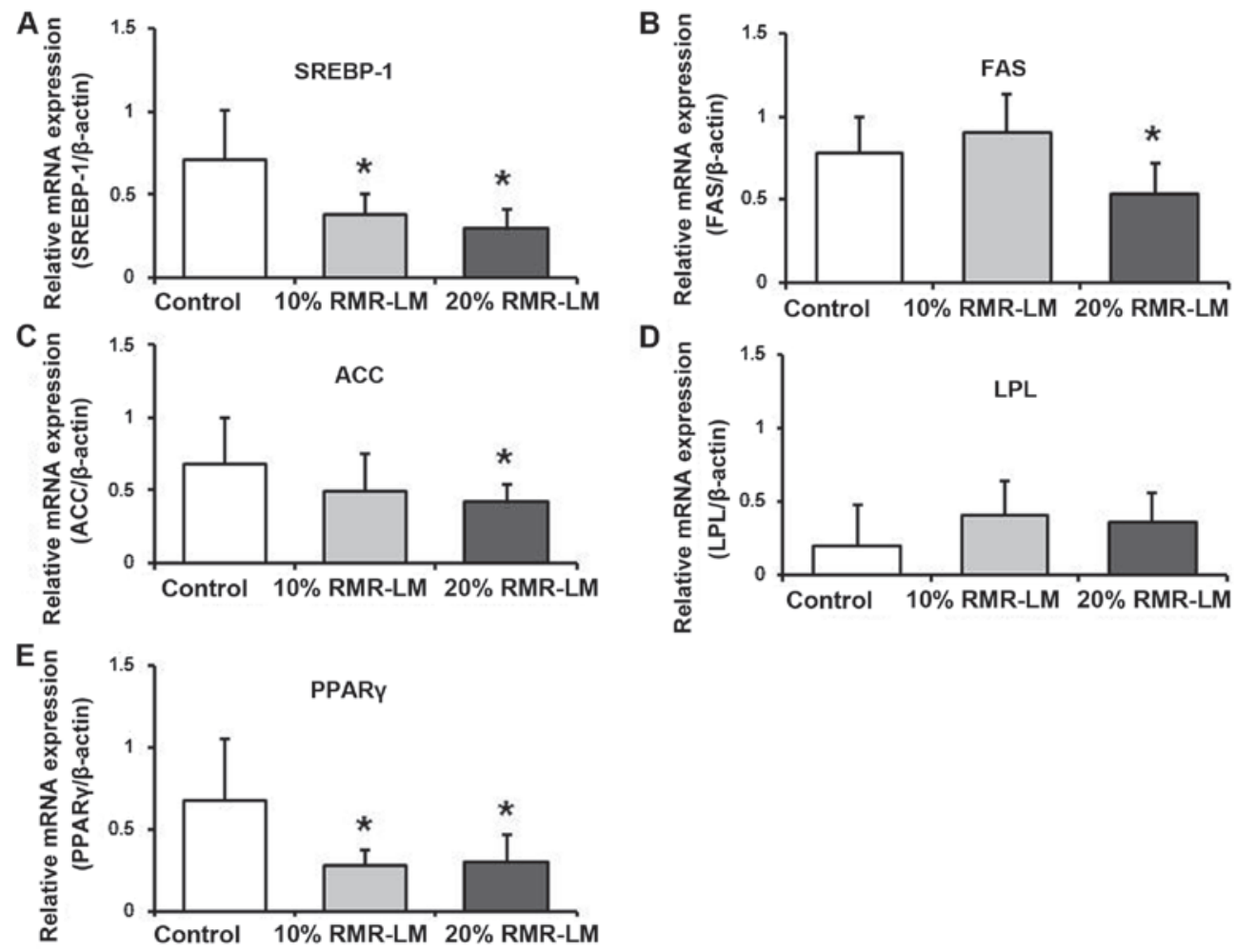

Figure 4. Effects of RMR-LM on relative mRNA expression in the liver. (A) sterol regulation element-binding protein (SREBP)-1; (B) fatty acid synthase (FAS); (C) acetyl-CoA carboxylase (ACC); (D) lipoprotein lipase (LPL); (E) peroxisome proliferator-activated receptor $\gamma$ (PPAR $\gamma$ ). $\beta$-actin was used as an internal control. Data are expressed as the mean \pm standard deviation ( $\mathrm{n}=10$ per group). $\mathrm{P}<0.05$ vs. control.

weight changes, reduction in total visceral fat weights, blood glucose level effects, and intraperitoneal glucose tolerance test results (14).
Obesity is associated with an increased risk of morbidity and mortality. It is also recognized as a major risk factor for metabolic diseases, lipid disorders, type-2 diabetes, and 
hypertension. The accumulation of fat in the body is a major characteristic of obesity $(15,16)$. To test whether the body weight reduction was caused by a decrease in adiposity, fat pads were dissected and weighed (17). The total visceral fat weight in the $10 \%$ RMR-LM and 20\% RMR-LM groups was significantly ( $\left.{ }^{*} \mathrm{P}<0.05\right)$ lower than that in the control group. The amount of fat mass is increased when the size of adipocytes are multiplied by proliferation and differentiation (17). The adipocyte size was calculated by dividing the number of adipocytes by the area counted. The adipocyte size in the 20\% RMR-LM group was significantly smaller than in the control group. An increased adipocyte number led to a decreased adipocyte size that the 20\% RMR-LM treatment had an anti-obesity effect.

While the LDL cholesterol are mainly attached to the arterial vessel wall causing arteriosclerosis, or the higher the plasma concentration as a factor that is the cause of heart disease, the risk is increased, HDL cholesterol is to go to the liver to remove the low molecular cholesterol that causes arteriosclerosis in the blood is known as a good cholesterol (7). Although the $20 \%$ RMR-LM group exhibited reductions in body weight, and total visceral fat weight, the serum TG level was not significantly different among the groups. The serum AST level and ALT level in the 10\% RMR-LM group or $20 \%$ RMR-LM group were lower than those in the control group, indicating that the RMR-LM treatments did not cause hepatic toxicity. And, the serum TC level in the 10\% RMR-LM group and 20\% RMR-LM group was lower than in the control group, but this difference was not significant (data not shown). These findings provided direct evidence that the RMR-LM extract had beneficial lipolytic effects on high-fat diet-induced obesity.

In our experiments, the significant decreases in body weight in the $10 \%$ RMR-LM group and 20\% RMR-LM group suggested that the RMR-LM extracts may reduce the visceral fat weight. The adipocyte size in the $20 \%$ RMR-LM group was significantly smaller than in the control group, indicating that the $20 \%$ RMR-LM treatment up-regulated lipolysis in adipocytes. Significant improvements in glucose tolerance were observed in the 10\% RMR-LM and 20\% RMR-LM groups compared with the control group. These results suggested that the 10\% RMR-LM and 20\% RMR-LM treatments had a hypoglycemia effect.

To clarify the mechanism on anti-obesity effect of the RMR-LM extracts, expression levels of genes related to lipid and fatty acid metabolism in the liver were investigated by real-time PCR. PPAR $\gamma$ is a key nuclear receptor transcription factor in adipogenesis and lipogenesis (18) and plays an important role in liver lipid storage and the differentiation of fat cells $(19,20)$. The findings in the present study indicated that both the 10\% RMR-LM group and 20\% RMR-LM group had significant $\left({ }^{*} \mathrm{P}<0.05\right)$ down-regulation of PPAR $\gamma$ mRNA expression in the liver. The expression of several lipogenic genes is regulated by SREBP-1 at the transcriptional level (21). Our evidence suggests that the 20\% RMR-LM extract may decrease lipogenesis partly by regulating PPAR $\gamma$ signaling. SREBP-1 is a major transcription factor involved in the activation of lipogenic genes such as FAS and ACC $(22,23)$. As the hepatic SREBP-1 mRNA level was significantly lower in the $10 \%$ RMR-LM group and 20\% RMR-LM group than in the control group, this lower SREBP-1 level could have contributed to the reduction in the FAS and ACC mRNA levels in the liver. The liver expression levels of FAS and ACC were significantly $\left({ }^{*} \mathrm{P}<0.05\right)$ suppressed in the mice in the $20 \%$ RMR-LM group. The 20\% RMR-LM extract was shown to be beneficial by lowering the body weight and visceral fat weight and preventing hepatic lipid accumulation. The reduction in the hepatic mRNA level of genes related to lipid anabolism such as SREBP-1 and PPAR $\gamma$ contributes to the anti-obesity effect of $20 \%$ RMR-LM.

In conclusion, robusta was cultivated first with $M$. ruber and then fermented with L. mesenteroides (RMR-LM). Significant improvements in glucose tolerance were observed in the $10 \%$ RMR-LM and 20\% RMR-LM groups compared with the control group. The serum glucose level was significantly lower in the 20\% RMR-LM group than in the groups. These results suggest that the $20 \%$ extract of RMR-LM may be a promising dietary supplement and food additive for inhibition of obesity and amelioration of diabetes and lipid metabolism imbalances.

\section{Acknowledgements}

This study was financially supported by the 'Animal Pharmaceutical Industry Support Hub' through the Ministry of Trade, Industry and Energy (MOTIE) and Korea Institute for Advancement of Technology (KIAT).

\section{References}

1. Lin S, Thomas TC, Storlien LH and Huang XF: Development of high fat diet-induced obesity and leptin resistance in C57Bl/6J mice. Int J Obes Relat Metab Disord 24: 639-646, 2000.

2. Li Z, Maglione M, Tu W, Mojica W, Arterburn D, Shugarman LR, Hilton L, Suttorp M, Solomon V, Shekelle PG and Morton SC: Meta-analysis: Pharmacologic treatment of obesity. Ann Intern Med 142: 532-546, 2005.

3. Tan S, Li M, Ding X, Fan S, Guo L, Gu M, Zhang Y, Feng L, Jiang D, Li Y, et al: Effects of Fortunella margarita fruit extract on metabolic disorders in high-fat diet-induced obese C57BL/6 mice. PLoS One 9: e93510, 2014.

4. Choudhary M and Grover K: Development of functional food products in relation to obesity. Funct Foods Health Dis 2: 188-197, 2012.

5. Kim H, Jeong JH, Shin JY, Kim DG and Yu KW: Immunomodulatory and anti-inflammatory activity of mulberry (Morus alba) leaves fermented with Hericium erinaceum mycelium by solid-state culture. J Korean Soc Food Sci Nutr 40: 1333-1339, 2011.

6. Peng CH, Liu LK, Chuang CM, Chyau CC, Huang CN and Wang CJ: Mulberry water extracts possess an anti-obesity effect and ability to inhibit hepatic lipogenesis and promote lipolysis. J Agric Food Chem 59: 2663-2671, 2011.

7. Sung J, Shin JY, Kim H, Baek GH, Yu KW, Yeon J and Lee J: Anti-obesity and anti-hyperlipidemic activities of fermented coffee with Monascus ruber mycelium by solid-state culture of green coffee beans. J Korean Soc Food Sci Nutr 43: 341-348, 2014.

8. Yang BK, Park JB and Song CH: Hypolipidemic effect of an exo-biopolymer produced from a submerged mycelial culture of Hericium erinaceus. Biosci Biotechnol Biochem 67: 1292-1298, 2003.

9. Hiwatashi K, Kosaka Y, Suzuki N, Hata K, Mukaiyama T, Sakamoto K, Shirakawa H and Komai M: Yamabushitake mushroom (Hericium erinaceus) improved lipid metabolism in mice fed a high-fat diet. Biosci Biotechnol Biochem 74: 1447-1451, 2010.

10. Vendruscolo F, Müller BL, Moritz DE, de Oliveira D, Schmidell W and Ninow JL: Thermal stability of natural pigments produced by Monascus ruber in submerged fermentation. Biocata Agric Biotechnol 2: 278-284, 2013.

11. Lim Y, Shin JY, Kim H, Baek GH, Yu KW, Jeong HS and Lee J: Anti-adipogenic effect of fermented coffee with Monascus ruber mycelium by solid-state culture of greens. J Korean Soc Food Sci Nutr 43: 624-629, 2014. 
12. Panda BP, Javed S and Ali M: Optimization of fermentation parameters for higher lovastatin production in red mold rice through co-culture of Monascus purpureus and Monascus ruber. Food Bioprocess Technol 3: 373-378, 2010.

13. Yang HS, Choi YJ, Oh HH, Moon JS, Jung HK, Kim KJ, Choi BS Lee JW and Huh CK: Antioxidative activity of mushroom water extracts fermented by lactic acid bacteria. J Korean Soc Food Sci Nutr 43: 80-85, 2014.

14. Murase T, Nagasawa A, Suzuki J, Hase T and Tokimitsu I: Beneficial effects of tea catechins on diet-induced obesity: Stimulation of lipid catabolism in the liver. Int J Obes Relat Metab Disord 26: 1459-1464, 2002.

15. Lee JR, Shin JH, Byun SH, Park SJ, Jo MJ, Park SM, Ku SK and Kim SC: Anti-obese and hypolipemic effects of the aqueous extracts of Raphani semen in mice fed high fat diet. J Korean Soc Appl Biol Chem 52: 50-57, 2009.

16. Poirier B, Bidouard JP, Cadrouvele C, Marniquet X, Staels B, O'Connor SE, Janiak P and Herbert JM: The anti-obesity effect of rimonabant is associated with an improved serum lipid profile. Diabetes Obes Metab 7: 65-72, 2005.

17. Choi H, Eo H, Park K, Jin M, Park EJ, Kim SH, Park JE and Kim S: A water-soluble extract from Cucurbita moschata shows anti-obesity effects by controlling lipid metabolism in a high fat diet-induced obesity mouse model. Biochem Biophys Res Commun 359: 419-425, 2007.
18. Lu Y, Xi W, Ding X, Fan S, Zhang Y, Jiang D, Li Y, Huang C and Zhou Z: Citrange fruit extracts alleviate obesity-associated metabolic disorder in high-fat diet-induced obese C57BL/6 mouse. Int J Mol Sci 14: 23736-23750, 2013.

19. Park MY, Lee KS and Sung MK: Effects of dietary mulberry, Korean red ginseng, and banaba on glucose homeostasis in relation to PPAR-alpha, PPAR-gamma, and LPL mRNA expressions. Life Sci 77: 3344-3354, 2005.

20. Tontonoz P, Hu E and Spiegelman BM: Stimulation of adipogenesis in fibroblasts by PPAR $\gamma 2$, a lipid-activated transcription factor. Cell 79: 1147-1156, 1994.

21. Murase T, Misawa K, Minegishi Y, Aoki M, Ominami H, Suzuki Y, Shibuya Y and Hase T: Coffee polyphenols suppress diet-induced body fat accumulation by downregulating SREBP-1c and related molecules in C57BL/6J mice. Am J Physiol Endocrinol Metab 300: E122-E133, 2011.

22. Huang HL, Hong YW, Wong YH, Chen YN, Chyuan JH, Huang CJ and Chao PM: Bitter melon (Momordica charantia L.) inhibits adipocyte hypertrophy and down regulates lipogenic gene expression in adipose tissue of diet-induced obese rats. Br J Nutr 99: 230-239, 2008

23. Lee YS, Cha BY, Yamaguchi K, Choi SS, Yonezawa T, Teruya T, Nagai K and Woo JT: Effects of Korean white ginseng extracts on obesity in high-fat diet-induced obese mice. Cytotechnology 62: 367-376, 2010 . 